\title{
THE SCALE HEIGHT OF BLUE HALO SUBDWARF B STARS
}

\author{
K.S. DE BOER, A. THEISSEN, U. HEBER AND S. MOEHLER \\ Sternwarte der Univ. Bonn, D-53121 Bonn, and \\ Remeis Sternw. U. Erlangen-Nürnberg, D-96049 Bamberg, and \\ Landessternwarte, Königstuhl, D-69117 Heidelberg
}

Numerous blue halo stars have been discovered during the past decade (e.g. the Palomar-Green Survey). Many are horizontal branch type, being HBA, HBB, sdB, or sdO star. The sdB stars $\left(\mathrm{T}_{\text {eff }}\right.$ between $\approx 18000$ and $\approx 30000 \mathrm{~K}$ ) are the endproducts of evolution in the red-giant phase. This means that the spatial distribution of the sdB stars can be used to get insight in the population nature and in their spatial origin.

We have observed a large number $(>100)$ of these stars with photometry (uvby) and spectroscopy (Balmer lines) in order to determine their physical parameters. Reports on these observations can be found in Heber et al. (1984), Heber (1986), de Boer et al. (1988), and in the series of papers: I: Moehler et al. 1990a, II: Moehler, Heber \& de Boer 1990b, III: Dreizler et al. 1990, IV: Theissen et al. 1993, V: Theissen et al. 1995.

Since the sdB stars are the result of giant evolution and thought to have a mass of about $0.5 \mathrm{M}_{\odot}$, one can now use the basic relations for stellar structure to determine the luminosity and thus the distance. For the three fields studied the z-distribution can be described by an exponential distribution. The scale height determined for each field has some uncertainty, but collectively we find that $\mathrm{z}=220 \mathrm{pc}\left({ }_{-30}^{+50} \mathrm{pc}\right)$.

This indicates that the sdB stars are part of the older Population I.

\section{References}

de Boer K.S., Heber U., Richtler T. 1988, A\&A 202, 113

Dreizler S., Heber U., Moehler S., de Boer K.S., et al, 1990a, A\&A 235, 234 (Pap. III)

Heber U., 1986, A\&A 155, 33

Heber U., Hunger K., Jonas G., Kudritzki R.-P., 1984, A\&A 130, 119

Moehler S., Richtler T., de Boer K.S., Heber U., et al, 1990a, A\&AS 86, 53 (Paper I)

Moehler S., Heber U., de Boer K.S., 1990b, A\&A 239, 265 (Paper II)

Theissen A., Moehler S., Heber U., de Boer K.S., 1993, A\&A 273, 524 (Paper IV)

Theissen A., Moehler S., Heber U., Schmidt J.H.K., de Boer K.S., 1995, A\&A (Paper V) 